\title{
Risk Factors and Clinical Outcomes of Neonatal Sepsis in Manado Tertiary Referral Hospital: A Single-center Study
}

\author{
Rocky Wilar*(iD, Hesti Lestari (D) \\ Department of Pediatrics, Sam Ratulangi University, RSUP Prof Dr. R. D. Kandou, Manado, Indonesia
}

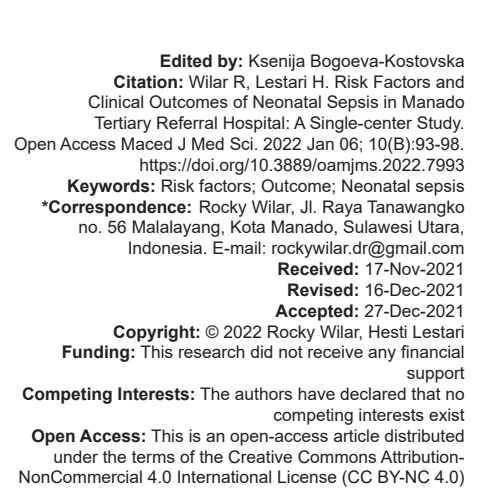

Introduction

Neonatal sepsis is defined as a clinical syndrome arising from the invasion of microorganisms into the bloodstream, which is occurred within the $1^{\text {st }}$ month of life [1].] It remains a major cause of morbidity and mortality among both term and preterm infants [2]. The World Health Organization (WHO) has estimated 4 million neonatal deaths occurred in the first 4 weeks of life, with a quarter of them were due to neonatal sepsis (incidence 42\%) [3]. In Indonesia, according to the Indonesian Basic Health Research (Riskesdas) in 2013, incidence of neonatal sepsis was $11 \%$, with several national journals reported mortality rate in tertiary and secondary referral hospitals were varied between regions [4]. A 2009 study at Indonesian main tertiary referral hospital, Cipto Mangunkusumo Hospital (RSCM), Jakarta, reported the incidence of neonatal sepsis of 98 cases/1000 live births. Meanwhile, another previous study in 2013 at Kandou General Hospital Manado, a tertiary referral hospital in Eastern Indonesia, reported mortality rate of $30.1 \%$ in neonatal sepsis (127 out of 421 neonates) [5].
Risk factors associated with neonatal sepsis can be divided into two main groups, such as neonatalrelated and maternal-related risk factors. The previous studies shown neonatal-related risk factors in neonatal sepsis were gender, birth weight, Apgar score at birth, gestational age, and location of delivery. Meanwhile, maternal-related risk factors include educational level, socio-economic status (SES), frequencies of antenatal care (ANC), mode of delivery, history of premature rupture of membrane (PROM) more than $12 \mathrm{~h}$, history of urinary tract infection (UTI) in pregnancy, and multiple gestations [6], [7].

In neonatal sepsis, clinical symptoms may rapidly progress from mild symptoms into death in $<24 \mathrm{~h}$, with various manifestations such as respiratory distress (tachypnea), vomiting, diarrhea, suckling refusal, hypothermia or hyperthermia, hypoglycemia or hyperglycemia, jaundice, lethargy, irritability, convulsions, prominent fontanelle, and shock, to disseminated intravascular coagulopathy (DIC). These symptoms are sometimes quite subtle in the early progression, thus early diagnosis and prompt treatment are necessary to improve outcomes and prevent mortality [8]. However, even with effective treatment, 
this condition causes inflated health-care cost due to prolonged length of stay (LOS). Thus, this study was conducted to evaluate risk factors and their association with clinical outcomes of neonatal sepsis at Kandou General Hospital Manado.

\section{Methods}

A single-center retrospective cohort study was conducted in Neonatal Intensive Care Unit (NICU) of Kandou General Hospital Manado, Indonesia. It is currently one of the largest tertiary referral teaching hospitals in Eastern Indonesia. Data were collected from medical records of all neonates with neonatal sepsis between January 2016 and December 2018. This study was approved by the Research Ethics Committee of Kandou General Hospital Manado.

Risk factors were divided into two groups, such as neonatal-related and maternal-related risk factors. Neonatal-related risk factors are as follows: Gender, birth weight, presence of hyperthermia, hypothermia, tachycardia (heart rate $>160 \mathrm{bpm}$ ), prolonged capillary refill time (CRT $\geq 3 \mathrm{~s}$ ), sclerema (infant panniculitis of skin and subcutaneous adipose tissue) [9], seizure, hypoglycemia, leukocyte count (leukocytosis $>25,000 / \mathrm{mm}^{3}$; leukopenia $<5000 / \mathrm{mm}^{3}$ ), thrombocytopenia (platelet count $<100.000 / \mathrm{mm}^{3}$ ), elevation of immature to total neutrophil ratio (I/T ratio $\geq 0.2$ ), and elevation of $\mathrm{C}$-reactive protein (CRP $\geq 6 \mathrm{mg} / \mathrm{L})$.

Maternal-related risk factors are as follows: Educational level, socio-economic status (based on National Health Insurance class I/high; II/middle; III/ low), antenatal care frequency (ANC $<4$ times and $\geq 4$ times), history of PROM ( $\geq 12 \mathrm{~h}$ ), history of urinary tract infection, mode of delivery, history of vaginal discharge/chorioamnionitis, multiple gestation, $1^{\text {st }} \mathrm{min}$ Apgar score (low Apgar <7, and normal Apgar >7), gestational age (term and preterm), and delivery location (in-hospital and non-hospital).

Clinical outcomes analyzed were survival outcome (discharged or death) and hospital LOS. LOS is defined as the number of hospital admission days before discharge. Participants with incomplete data and congenital anomalies were excluded from the study.

All collected data were analyzed univariately, according to the each risk factor and its outcome. All risk factors with $p<0.2$ in univariate model were further analyzed using multivariate model to look for significant association with outcome after adjusted to all other variables. Statistical analysis used in this study is logistic regression, Mann-Whitney, and Kruskal-Wallis test, using SPSS version 25 for Windows.

\section{Results}

A total of 365 subjects (216 males and 149 females) with neonatal sepsis met the inclusion criteria. Almost half of the participants (47.1\%) weighed $<2500 \mathrm{~g}$ at birth. Based on sepsis clinical features, 46 (12.6\%) had hyperthermia, 29 (7.9\%) had hypothermia, $267(73.1 \%)$ were presented with tachycardia, 219 (60\%) with tachypnea, $163(61.5 \%)$ with prolonged CRT, and 125 (34.2\%) with hypoglycemia. Other subtle clinical manifestations of neonatal sepsis were also observed, such as fussiness (50.9\%), suckling refusal $(72.8 \%)$, and inactivity $(2.4 \%)$. A small percentage of neonates $(7 \%)$ were presented with convulsion during hospitalization. Sclerema was observed in $71(19.4 \%)$ subjects. Based on laboratory parameters, 157 (43.1\%) had leukopenia, 62 (16.9\%) had leukocytosis, and $212(58 \%)$ had thrombocytopenia. More than half of participants showed elevated $\mathrm{I} / \mathrm{T}$ ratio and CRP (55.3\% and 63.8\%, respectively) (Table 1).

When analyzed with survival outcome, univariate analysis of neonatal-related risk factors showed several risk factors likely associated, such as hyperthermia, hypothermia, tachypnea, hypoglycemia, convulsion, leukopenia, thrombocytopenia, and sclerema. However, after adjusted in multivariate analysis, only two factors were associated, namely, tachypnea (OR 4.94; $\mathrm{p}<0.001)$ and sclerema (OR 34.47; $p<0.001$ ) (Table 2).

Regarding maternal-related risk factors, almost half $(44.4 \%)$ were high-school and university graduate, with majority of mothers $(57.8 \%)$ had low SES. The majority $(61 \%)$ of mothers only received their ANC follow-up <4 times during pregnancy. More than half $(61.09 \%)$ had a history of PROM. About a third of all mothers had a history of pregnancy UTI and chorioamnionitis $(35.9 \%$ and $37 \%$, respectively). About 225 (61.6\%) delivered in-hospital location and 215 (58.9\%) delivered by caesarean section procedure. Majority were term at birth $(72.3 \%)$, with $65.7 \%$ of all newborns had low $1^{\text {st }}$ min Apgar score $<7$. Nineteen mothers $(5.2 \%)$ had multiple gestations (Table 1$)$.

Univariate analysis of maternal-related risk factors showed that low SES and low Apgar score likely associated with survival outcome. However, after adjusted in multivariate analysis, none of these factors were significantly associated with survival outcome (Table 3).

Among those who survived (268 subjects), when analyzed with LOS outcome, from neonatalrelated risk factors showed only very low birth weight significantly associated with prolonged LOS (median 32 days; $p<0.001$ ) (Table 4). On maternal-related risk factors, several risk factors were significantly associated with LOS, such as PROM ( $p=0.009)$, preterm gestational age $(p<0.001)$, and non-hospital delivery location $(p=0.013)$ (Table 5). 
Wilar and Lestari. Risk factors and clinical outcomes of neonatal sepsis in Manado

Table 1: Risk factors characteristics and survival rates in neonatal sepsis

\begin{tabular}{|c|c|c|c|c|}
\hline Risk factors & Disch & & Dea & \\
\hline & $\mathrm{n}$ & $\%$ & $\mathrm{n}$ & $\%$ \\
\hline Neonatal-related & & & & \\
\hline Gender & & & & \\
\hline Male & 160 & 74,1 & 56 & 25,9 \\
\hline Female & 108 & 72.5 & 41 & 27.5 \\
\hline Birth weight & & & & \\
\hline Very low & 20 & 71.4 & 8 & 28.6 \\
\hline Low & 106 & 73.6 & 38 & 26.4 \\
\hline Normal & 142 & 73.6 & 51 & 26.4 \\
\hline Hyperthermia & & & & \\
\hline Yes & 29 & 63.0 & 17 & 37.0 \\
\hline No & 239 & 74.9 & 80 & 25.1 \\
\hline Hypothermia & & & & \\
\hline Yes & 10 & 34.5 & 19 & 65.5 \\
\hline No & 258 & 76.8 & 78 & 23.2 \\
\hline Tachycardia & & & & \\
\hline Yes & 194 & 72.7 & 73 & 27.3 \\
\hline No & 74 & 75.5 & 24 & 24.5 \\
\hline Tachypnea & & & & \\
\hline Yes & 139 & 63.5 & 80 & 36.5 \\
\hline No & 129 & 88.4 & 17 & 11.6 \\
\hline Prolonged CRT & & & & \\
\hline Yes & 121 & 74.2 & 42 & 25.8 \\
\hline No & 147 & 72.8 & 55 & 27.2 \\
\hline Fussiness & & & & \\
\hline Yes & 135 & 72.6 & 51 & 27.4 \\
\hline No & 133 & 74.3 & 46 & 25.7 \\
\hline Hypoglycemia & & & & \\
\hline Yes & 86 & 68.8 & 39 & 31.2 \\
\hline No & 182 & 75.8 & 58 & 24.2 \\
\hline Suckling refusal & & & & \\
\hline Yes & 198 & 74.4 & 68 & 25.6 \\
\hline No & 70 & 70.7 & 29 & 29.3 \\
\hline Inactivity & & & & \\
\hline Yes & 47 & 74.6 & 16 & 25.4 \\
\hline No & 221 & 73.2 & 81 & 26.8 \\
\hline Convulsion & & & & \\
\hline Yes & 20 & 87.0 & 3 & 13.0 \\
\hline No & 248 & 72.5 & 94 & 27.5 \\
\hline Leukopenia & & & & \\
\hline Yes & 127 & 80.9 & 30 & 19.1 \\
\hline No & 141 & 67.8 & 67 & 32.2 \\
\hline Leukocytosis & & & & \\
\hline Yes & 44 & 71.0 & 18 & 29.0 \\
\hline No & 224 & 73.9 & 79 & 26.1 \\
\hline Thrombocytopenia & & & & \\
\hline Yes & 165 & 77.8 & 47 & 22.2 \\
\hline No & 103 & 67.3 & 50 & 32.7 \\
\hline Elevated I/T ratio & & & & \\
\hline Yes & 124 & 76.1 & 39 & 23.9 \\
\hline No & 144 & 71.3 & 58 & 28.7 \\
\hline Elevated CRP & & & & \\
\hline Yes & 94 & 71.2 & 38 & 28.8 \\
\hline No & 174 & 74.7 & 59 & 25.3 \\
\hline Sclerema & & & & \\
\hline Yes & 12 & 16.9 & 59 & 83.1 \\
\hline No & 256 & 87.1 & 38 & 12.9 \\
\hline Maternal-related & & & & \\
\hline Educational level & & & & \\
\hline Elementary & 57 & 65.5 & 30 & 34.5 \\
\hline Middle school & 87 & 75 & 29 & 25 \\
\hline High school & 80 & 79.2 & 21 & 20.8 \\
\hline University graduate & 44 & 72,1 & 17 & 27.9 \\
\hline SES & & & & \\
\hline Low & 148 & 70.1 & 63 & 29,9 \\
\hline Middle & 68 & 75.6 & 22 & 24,4 \\
\hline High & 52 & 81.2 & 12 & 18,8 \\
\hline ANC frequency & & & & \\
\hline$<4$ times & 160 & 71.7 & 63 & 28.3 \\
\hline$\geq 4$ times & 108 & 76.7 & 34 & 23.9 \\
\hline History of PROM & & & & \\
\hline Yes & 166 & 74.4 & 57 & 25.6 \\
\hline No & 102 & 71.8 & 40 & 28.2 \\
\hline History of UTI & & & & \\
\hline Yes & 98 & 74.8 & 33 & 25.2 \\
\hline No & 170 & 72.6 & 64 & 27.4 \\
\hline Chorioamnionitis & & & & \\
\hline Yes & 95 & 70.4 & 40 & 29.6 \\
\hline No & 173 & 75.2 & 57 & 24.8 \\
\hline Mode of delivery & & & & \\
\hline Caesarean section & 158 & 73.5 & 57 & 26.5 \\
\hline Vaginal birth & 110 & 73.3 & 40 & 26.7 \\
\hline Multiple gestations & & & & \\
\hline Yes & 13 & 68.4 & 6 & 31.6 \\
\hline $\begin{array}{l}\text { No } \\
\text { Low Apgar score }\end{array}$ & 255 & 73.7 & 91 & 26.3 \\
\hline Yes & 169 & 70.4 & 71 & 29.6 \\
\hline No & 97 & 78.9 & 26 & 21.1 \\
\hline Gestational age & & & & \\
\hline Preterm & 75 & 74.3 & 26 & 25.7 \\
\hline Term & 193 & 73.1 & 71 & 26.9 \\
\hline Delivery location & & & & \\
\hline Non-hospital & 103 & 73.6 & 37 & 26.4 \\
\hline In-hospital & 165 & 73.3 & 60 & 26.7 \\
\hline
\end{tabular}

Table 2: Survival outcome analysis on neonatal-related risk factors

\begin{tabular}{|c|c|c|c|c|}
\hline \multirow{2}{*}{ Risk factors } & \multicolumn{2}{|c|}{ Univariate } & \multicolumn{2}{|c|}{ Multivariate } \\
\hline & $p$ value & OR $(95 \% \mathrm{Cl})$ & $p$ value & OR $(95 \% \mathrm{Cl})$ \\
\hline \multicolumn{5}{|l|}{ Gender } \\
\hline Male & ref & Ref & & \\
\hline Female & 0.735 & $1.085(0.677-1.737)$ & & \\
\hline \multicolumn{5}{|l|}{ Birth weight } \\
\hline Very low & 0.81 & $1.114(0.462-2.685)$ & & \\
\hline Low & 0.994 & $0.998(0.612-1.629)$ & & \\
\hline Normal & ref & Ref & & \\
\hline Hyperthermia & 0.091 & $1.751(0.914-3.355)$ & 0.504 & $\begin{array}{l}1.378 \\
(0.538-3.531)\end{array}$ \\
\hline Hypothermia & $<0.001$ & $6.285(2.806-14.078)$ & 0.058 & $\begin{array}{l}2.848 \\
(0.967-8.391)\end{array}$ \\
\hline Tachycardia & 0.585 & $1.16(0.681-1.978)$ & & \\
\hline Tachypnea & $<0.001$ & $4.367(2.456-7.767)$ & $<0.001^{*}$ & $\begin{array}{l}4.947 \\
(2.257-10.841)\end{array}$ \\
\hline Prolonged CRT & 0.753 & $0.928(0.581-1.482)$ & & \\
\hline Fussiness & 0.71 & $1.092(0.686-1.739)$ & & \\
\hline Hypoglycemia & 0.149 & $1.423(0.88-2.3)$ & 0.389 & $\begin{array}{l}1.333 \\
(0.693-2.563)\end{array}$ \\
\hline Suckling refusal & 0.473 & $0.829(0.496-1.385)$ & & \\
\hline Inactivity & 0.816 & $0.929(0.499-1.73)$ & & \\
\hline Convulsion & 0.129 & $0.396(0.115-1.363)$ & 0.392 & $\begin{array}{l}0.5 \\
(0.102-2.452)\end{array}$ \\
\hline Leukopenia & 0.005 & $0.497(0.304-0.814)$ & 0.685 & $\begin{array}{l}1.233 \\
(0.449-3.384)\end{array}$ \\
\hline Leukocytosis & 0.631 & $1.16(0.633-2.125)$ & & \\
\hline Thrombocytopenia & 0.025 & $0.587(0.367-0.937)$ & 0.08 & $\begin{array}{l}0.413 \\
(0.154-1.111)\end{array}$ \\
\hline Elevated $\mathrm{I} / \mathrm{T}$ ratio & 0.304 & $1.281(0.799-2.052)$ & & \\
\hline Elevated CRP & 0.471 & $1.192(0.739-1.924)$ & & \\
\hline Sclerema & $<0.001$ & $33.123(16.317-67.24)$ & $<0.001^{*}$ & $\begin{array}{l}34.471 \\
(15.135-78.509)\end{array}$ \\
\hline
\end{tabular}

\section{Discussion}

Based on this study result, among neonatalrelated risk factors, tachypnea and sclerema were found to be significantly associated with mortality in neonatal sepsis. Infants with neonatal sepsis presented with tachypnea were 5 times more likely to develop mortality (OR 4.947, 95\% Cl 2.257-10.841; $p<0.001$ ). Tachypnea is considered as an indicator of pulmonary dysfunction and is commonly seen in pneumonia and acute respiratory distress syndrome (ARDS), both of which were risk factors associated with increased mortality in sepsis patients. Stimulation of primary

Table 3: Survival outcome analysis on maternal-related risk factors

\begin{tabular}{|c|c|c|c|c|}
\hline \multirow[t]{2}{*}{ Risk factors } & \multicolumn{2}{|c|}{ Univariate } & \multicolumn{2}{|c|}{ Multivariate } \\
\hline & $p$ value & OR $(95 \% \mathrm{Cl})$ & $p$ value & $\begin{array}{l}\text { OR } \\
(95 \% \mathrm{Cl})\end{array}$ \\
\hline \multicolumn{5}{|l|}{ Educational level } \\
\hline Elementary & 0.396 & $1.362(0.688-2.78)$ & & \\
\hline Middle school & 0.679 & $0.863(0.428-1.737)$ & & \\
\hline High school & 0.304 & $0.679(0.325-1.421)$ & & \\
\hline \multirow{2}{*}{\multicolumn{5}{|c|}{ SES }} \\
\hline & & & & \\
\hline Low & 0.084 & $1.845(0.922-3.69)$ & 0.381 & $\begin{array}{l}0.776 \\
(0.44-1.368)\end{array}$ \\
\hline Middle & 0.402 & $1.402(0.636-3.091)$ & 0.117 & $\begin{array}{l}0.572 \\
(0.285-1.15)\end{array}$ \\
\hline High & ref & ref & ref & ref \\
\hline ANC frequency $<4$ times & 0.364 & $1.251(0.771-2.028)$ & & \\
\hline History of PROM & 0.582 & $0.876(0.545-1.406)$ & & \\
\hline History of UTI & 0.654 & $0.894(0.549-1.457)$ & & \\
\hline Chorioamnionitis & 0.312 & $1.278(0.794-2.056)$ & & \\
\hline $\begin{array}{l}\text { Cesarean section } \\
\text { delivery }\end{array}$ & 0.974 & $0.992(0.619-1.59)$ & & \\
\hline Multiple gestations & 0.612 & $1.293(0.477-3.503)$ & & \\
\hline Low Apgar score & 0.085 & $0.638(0.382-1.067)$ & 0.108 & $\begin{array}{l}1.527 \\
(0.911-2.56)\end{array}$ \\
\hline Preterm gestational age & 0.824 & $0.942(0.559-1.589)$ & & \\
\hline $\begin{array}{l}\text { Non-hospital delivery } \\
\text { location }\end{array}$ & 0.822 & $1.058(0.649-1.725)$ & & \\
\hline
\end{tabular}


respiratory control center in medulla oblongata by bacterial endotoxins and other inflammatory mediators was also possible causes of tachypnea to develop. When tissue hypoperfusion occurs due to neonatal sepsis and shock, the respiratory rate also increases to compensate the ongoing metabolic acidosis [9], [10]. This is consistent with the previous study by Jumah et al. which found strong association between tachypnea and early onset neonatal sepsis/EONS $(p<0.01)$ [11]. Another study about predictor analysis of mortality in neonatal sepsis also found that newborns with respiratory distress were significantly associated with higher mortality (OR 3.4; $p=0.007$ ) [12].

Furthermore, this study also showed that neonatal sepsis infants presented with sclerema had 34 times the risk of dying than those without sclerema (OR 34.471, 95\% Cl 15.135-78.509; p < 0.001). The previous studies also reported similar result, in which, sclerema was strongly associated with mortality in newborns [12], [13], [14]. Sclerema is a type of panniculitis in infancy which manifests as hardening of the skin and subcutaneous adipose tissue extending

Table 4: LOS outcome analysis on neonatal-related risk factors

\begin{tabular}{|c|c|c|c|}
\hline Risk factors & $\mathrm{n}(\%)$ & Median LOS (IQR; days) & $p$ value \\
\hline \multicolumn{4}{|l|}{ Gender } \\
\hline Male & $160(59.7 \%)$ & $11(8-17)$ & \multirow[t]{2}{*}{$0.621^{\mathrm{a}}$} \\
\hline Female & $108(40.3 \%)$ & $12(8-16)$ & \\
\hline \multicolumn{4}{|l|}{ Birth weight } \\
\hline Very low & $20(7.5 \%)$ & $32(26-38)$ & \multirow[t]{3}{*}{$<0.001^{* b}$} \\
\hline Low & $106(39.6 \%)$ & $11(9-16)$ & \\
\hline Normal & $142(53 \%)$ & $10(8-14)$ & \\
\hline \multicolumn{4}{|c|}{ Hyperthermia } \\
\hline Yes & $29(10.8 \%)$ & $10(8-16)$ & \multirow[t]{2}{*}{$0.691^{\mathrm{a}}$} \\
\hline No & $239(89.2 \%)$ & $11(8-17)$ & \\
\hline \multicolumn{4}{|c|}{ Hypothermia } \\
\hline Yes & $10(3.7 \%)$ & $11(9-15)$ & \multirow[t]{2}{*}{$0.927^{\mathrm{a}}$} \\
\hline No & $258(96.3 \%)$ & $11(8-17)$ & \\
\hline \multicolumn{4}{|l|}{ Tachycardia } \\
\hline Yes & $194(72.4 \%)$ & $11(8-17)$ & \multirow[t]{2}{*}{$0.98^{\mathrm{a}}$} \\
\hline No & $74(27.6 \%)$ & $11(8-16)$ & \\
\hline \multicolumn{4}{|l|}{ Tachypnea } \\
\hline Yes & $139(51.9 \%)$ & $11(8-17)$ & \multirow[t]{2}{*}{$0.366^{\mathrm{a}}$} \\
\hline No & $129(48.1 \%)$ & $11(9-16)$ & \\
\hline \multicolumn{4}{|c|}{ Prolonged CRT } \\
\hline Yes & $121(45.1 \%)$ & $12(9-17)$ & \multirow[t]{2}{*}{$0.286^{\mathrm{a}}$} \\
\hline No & $147(54.9 \%)$ & $11(8-17)$ & \\
\hline \multicolumn{4}{|l|}{ Fussiness } \\
\hline Yes & $135(50.4 \%)$ & $11(9-17)$ & \multirow[t]{2}{*}{$0.584^{a}$} \\
\hline No & $133(49.6 \%)$ & $11(8-17)$ & \\
\hline \multicolumn{4}{|c|}{ Hypoglycemia } \\
\hline Yes & $86(32.1 \%)$ & $13(9-19)$ & \multirow[t]{2}{*}{$0.053^{a}$} \\
\hline No & $182(67.9 \%)$ & $11(8-16)$ & \\
\hline \multicolumn{4}{|c|}{ Suckling refusal } \\
\hline Yes & $198(73.9 \%)$ & $11(8-17)$ & $0.763^{\mathrm{a}}$ \\
\hline No & $70(26.1 \%)$ & $11(8-17)$ & \\
\hline Inactivity & & & \\
\hline Yes & $47(17.5 \%)$ & $10(8-17)$ & $0.568^{\mathrm{a}}$ \\
\hline No & $221(82.5 \%)$ & $11(8-17)$ & \\
\hline Convulsion & & & \\
\hline Yes & $20(7.5 \%)$ & $13(11-22)$ & $0.193^{\mathrm{a}}$ \\
\hline No & $248(92.5 \%)$ & $11(8-17)$ & \\
\hline Leukopenia & & & \\
\hline Yes & $127(47.4 \%)$ & $11(8-16)$ & $0.352^{\mathrm{a}}$ \\
\hline No & $141(52.6 \%)$ & $12(8-19)$ & \\
\hline Leukocytosi & & & \\
\hline Yes & $44(16.4 \%)$ & $12(9-17)$ & $0.687^{\mathrm{a}}$ \\
\hline No & $224(83.6 \%)$ & $11(8-17)$ & \\
\hline Thrombocyt & & & \\
\hline Yes & $165(61.6 \%)$ & $11(8-16)$ & $0.584^{\mathrm{a}}$ \\
\hline No & $103(38.4 \%)$ & $11(8-19)$ & \\
\hline Elevated $\mathrm{I} / \mathrm{T}$ & & & \\
\hline Yes & $144(53.7 \%)$ & $11(8-17)$ & $0.701^{\mathrm{a}}$ \\
\hline No & $124(46.3 \%)$ & $11(8-17)$ & \\
\hline Elevated $\mathrm{CF}$ & & & \\
\hline Yes & $174(64.9 \%)$ & $11(8-16)$ & $0.19^{\mathrm{a}}$ \\
\hline No & $94(35.1 \%)$ & $13(8-17)$ & \\
\hline Sclerema & & & \\
\hline Yes & $12(4.5 \%)$ & $14(10-32)$ & $0.097^{\mathrm{a}}$ \\
\hline No & $256(95.5 \%)$ & $11(8-17)$ & \\
\hline
\end{tabular}

throughout the body, and sparing the fat-free soles, palms, and genitalia [9]. This physical finding is always associated with septic shock, as most patients with sclerema would die within hours to days, even when the patient was admitted in intensive care unit. Septic shock itself is a strong predictor of death due to impaired and ineffective peripheral circulation [13].

This study also found significant association between birth weight and prolonged LOS in the NICU, in which neonatal sepsis infants with birth weight $<1500 \mathrm{~g}$ spent approximately 32 days in the NICU. A study by Fenny et al. reported LBW infants spent at least 4 additional days on the neonatal ward, when compared to normal birth weight [14]. In LBW infants, particularly those with prematurity, their vital organs maturation (liver, lungs, enzymes, digestion, brain, and immunity) not yet completed. Therefore, they are more prone to complications and require prolonged days of close monitoring in the NICU [15]. Another study conducted by Payne et al. reported that VLBW infants with blood stream infection (BSI) had prolonged mean LOS, ranging from 48 to 101 days [16]. Clinical threshold for determining discharge timing could also influence the LOS. Neonates are rarely discharged before they gain the ability to suck and feed (which usually seen in 35 weeks of gestational age and older infants), thus prematurity became the main reason for their prolonged LOS [17].

From univariate analysis of maternal-related risk factors, low SES and low Apgar score were likely associated (but not significant) with survival outcome of neonatal sepsis ( $p=0.084$ and $p=0.085$, respectively).

Table 5: LOS outcome analysis on maternal-related risk factors

\begin{tabular}{|c|c|c|c|}
\hline Risk factors & $\mathrm{n}(\%)$ & $\begin{array}{l}\text { Median LOS } \\
\text { (IQR; days) }\end{array}$ & $p$ value \\
\hline \multicolumn{4}{|l|}{ Educational level } \\
\hline Elementary & $57(21.3)$ & $10(16-7)$ & \multirow[t]{4}{*}{$0.095^{\mathrm{a}}$} \\
\hline Middle school & $87(32.5)$ & $11(16-8)$ & \\
\hline High school & $80(29.9)$ & $11(16-8)$ & \\
\hline University graduate & $44(16.4)$ & $14(21-10)$ & \\
\hline \multicolumn{4}{|l|}{ SES } \\
\hline Low & $148(55.2)$ & $11(17-8)$ & \multirow[t]{3}{*}{$0.476^{\mathrm{a}}$} \\
\hline Middle & $68(25.4)$ & $13(18-9)$ & \\
\hline High & $52(19.4)$ & $11(17-9)$ & \\
\hline \multicolumn{4}{|l|}{ ANC frequency } \\
\hline$<4$ times & $160(59.7)$ & $12(19-8)$ & \multirow{2}{*}{$0.23^{\mathrm{b}}$} \\
\hline$\geq 4$ times & $108(40.3)$ & $11(14-8)$ & \\
\hline \multicolumn{4}{|l|}{ History of PROM } \\
\hline Yes & $166(61.9)$ & $13(19-8)$ & \multirow{2}{*}{$0.009^{* b}$} \\
\hline No & $102(38.1)$ & $10(14-8)$ & \\
\hline \multicolumn{4}{|l|}{ History of UTI } \\
\hline Yes & $98(36.6)$ & $11(17-9)$ & \multirow[t]{2}{*}{$0.84^{b}$} \\
\hline No & $170(63.4)$ & $11(17-8)$ & \\
\hline \multicolumn{4}{|l|}{ Chorioamnionitis } \\
\hline Yes & $95(35.4)$ & $10(15-8)$ & \multirow[t]{2}{*}{$0.182^{b}$} \\
\hline No & $173(64.6)$ & $12(17-8)$ & \\
\hline \multicolumn{4}{|l|}{ Mode of delivery } \\
\hline Caesarean section & $158(59)$ & $10(17-8)$ & \multirow[t]{2}{*}{$0.173^{\mathrm{b}}$} \\
\hline Vaginal birth & $110(41)$ & $12(17-9)$ & \\
\hline \multicolumn{4}{|l|}{ Multiple gestations } \\
\hline Yes & $13(4.9)$ & $14(17-9)$ & \multirow[t]{2}{*}{$0.422^{\mathrm{b}}$} \\
\hline No & 255 (95.1) & $11(17-8)$ & \\
\hline \multicolumn{4}{|l|}{ Low Apgar score } \\
\hline$<7$ & $169(63.5)$ & $11(17-8)$ & \multirow[t]{2}{*}{$0.865^{\mathrm{b}}$} \\
\hline$>7$ & $97(36.5)$ & $11(16-8)$ & \\
\hline \multicolumn{4}{|l|}{ Gestational age } \\
\hline Preterm & $75(28)$ & $15(23-11)$ & \multirow[t]{2}{*}{$<0.001^{* \text { * }}$} \\
\hline Term & $193(72)$ & $10(15-8)$ & \\
\hline \multicolumn{4}{|l|}{ Delivery location } \\
\hline Non-hospital & $103(38.4)$ & $12(19-9)$ & \multirow[t]{2}{*}{$0.013^{* b}$} \\
\hline In-hospital & $165(61.6)$ & $10(17-7)$ & \\
\hline
\end{tabular}


The previous study by Onyedibe et al. reported low socio-economic factor associated with the higher incidence of neonatal sepsis (OR 2.27; $p=0.047$ ), due to poor hygiene and sanitation, limited access to safe water sources, and limited access to antenatal care as contributing factors [18]. In regards to low Apgar score, our finding is consistent with Hayun et al., who reported that neonates with low Apgar score were 14.05 times at risk of developing EONS compared to neonates with good Apgar score at birth [19]. Apgar score is widely known and used as the earliest tool to assess neonatal asphyxia [20]. Low $1^{\text {st }}$ min Apgar score increases the need for mechanical ventilation and umbilical catheter insertion, in which both procedures expose neonates to sepsis causative microbes [21]. Furthermore, aggressive neonatal resuscitation procedure due to asphyxia could cause damage to respiratory tract and serves as another entry point for pathogenic germs [22].

Among maternal-related risk factors, several factors were significantly associated with prolonged LOS, such as PROM ( $p=0.009)$, gestational age $(p<0.001)$, and delivery location $(p=0.013)$. History of PROM and delivery location is well-known risk factors of perinatal infection and neonatal sepsis. Infection by itself is an independent risk factor known to affect LOS in the NICU. Rozanska et al. reported that occurrence of more than one clinical infection was associated with prolonged LOS [23].

To the best of our knowledge, there was no previously published study in Indonesia evaluating risk factors of neonatal sepsis and their association with clinical outcomes. This study showed that in neonatal sepsis, the presence of tachypnea and sclerema was significant risk factors for mortality. Meanwhile, premature rupture of the membrane, very low birth weight, preterm gestational age, and non-hospital delivery location were risk factors associated with prolonged length of stay.

However, this study was hampered by several limitations. Due to its retrospective nature and singlecentered, results might not be representative to the whole population. Lack of proper documentation also prevented some subjects to be included in the study. In addition, this study did not differentiate the exact onset of clinical symptoms in subjects, which might create further potential biases and confounding factors. Therefore, further studies with different population characteristics, larger number of subjects, and better methods are much needed.

\section{References}

1. Shakya A, Shresta D, Shakya H, Shah SC, Dhakal AK. Clinical profile and outcome of neonates admitted to the neonatal care unit at a teaching hospital in Lalipur, Nepal. J Kathmandu Med
Col. 2014;3(4):144-8. https://doi.org/10.3126/jkmc.v3i4.13370

2. Malik S, Gohiya P, Khan IA. Morbidity profile and mortality of neonates admitted in neonatal intensive care unit of a Central India teaching institute: A prospective observational study. J Clin Neonatol. 2016;5(3):168-73. https://doi. org/10.4103/2249-4847.191251

3. Lawn JE, Cousens S, Zupan J, Lancet Neonatal Survival Steering Team. 4 million neonatal deaths: When? Where? Why? Lancet. 2005;365(9462):891-900. https://doi.org/10.1016/ S0140-6736(05)71048-5

PMid:15752534

4. Kementerian Kesehatan RI. Riset Kesehatan Dasar (Riskesdas); 2013. Available from: https://www.litbang.kemkes. go.id/laporan-riset-kesehatan-dasar-riskesdas [Last accessed on 2020 June 15].

5. Prof Dr. R. D. Kandou. Profil RSUP Dr. R. D. Kandou Manado; 2020. Available from: https://www.rsupkandou.com/profil [Last accessed on 2020 Jun 15].

6. Widayati K, Kurniati DP, Windiani GA. Risk factors of neonatal sepsis at perinatology unit Sanglah general public hospital Denpasar. Public Health Prev Med Arch. 2016;4(1):67-73. https://doi.org/10.15562/phpma.v4i1.59

7. Adatara P, Afaya A, Salia SM. Risk factors associated with neonatal sepsis: A case study at a specialist hospital in Ghana. ScientificWorldJournal. 2019;2019:9369051. https://doi. org/10.1155/2019/9369051 PMid:30692878

8. Rennie JM. Roberton's Textbook of Neonatology. Infection in the Newborn. $5^{\text {th }}$ ed. Philadelphia, PA: Elsevier Churchill Livingstone; 2012. p. 1013-64.

9. Gomella TC, Cunningham MD, Eyal FG, Tuttle DJ. Neonatology Management, Procedures, on-Call Problem, Diseases, and Drugs. Neonatal Sepsis. $7^{\text {th }}$ ed. New York: McGraw-Hill Education; 2013. p. 427-36.

10. Goldstein B, Giroir B, Randolph A. International consensus conference on pediatric sepsis. International pediatric consensus conference: Definitions for sepsis and organ dysfunction in pediatrics. Pediatr Crit Care Med. 2005;6(1):2-8. https://doi.org/10.1097/01.PCC.0000149131.72248.E6 PMid:15636651

11. Jumah DS, Hassan MD. Predictors of mortality outcome in neonatal sepsis. Med J Basrah Univ. 2007;25(1):11-8. https:// doi.org/10.33762/mjbu.2007.48118

12. Ogunlesi TA, Ogunfowora OB. Predictors of mortality in neonatal septicemia in an underresourced setting. J Natl Med Assoc. 2010;102(10):915-21. https://doi.org/10.1016/ s0027-9684(15)30710-0

PMid:21053706

13. Chisti MJ, Saha S, Roy CN, Ahmed T, Faruque AS, Salam MA, et al. Predictors of mortality in infants with sclerema presenting to the centre for diarrhoeal disease, Dhaka. Ann Trop Paediatr. 2009;29(1):45-50. https://doi.org/10.1179/146532809X402024 PMid:19222934

14. Fenny AP, Otieku E, Labi K, Asante FA, Enemark U. Costs and extra length of stay because of Neonatal Bloodstream Infection at a Teaching Hospital in Ghana. Pharmacoeconomics. 2021;5(1):111-20. https://doi.org/10.1007/s41669-020-00230-x PMid:32940852

15. Belachew A, Tewabe T. Neonatal sepsis and its association with birth weight and gestational age among admitted neonates in Ethiopia: systematic review and meta-analysis. BMC Pediatr. 2020;20:55. https://doi.org/10.1186/s12887-020-1949-x

16. Payne N, Carpenter JH, Badger GJ, Horbar JD, Rogowski J. Marginal increase in cost and excess length of stay associated with nosocomial bloodstream infections in surviving very low 
birth weight infants. Pediatrics. 2004;114(2):348-55. https://doi. org/10.1542/peds.114.2.348

PMid:15286215

17. Seaton SE, Barker L, Jenkins D, Draper ES, Abrams KR, Manktelow BN. What factors predict length of stay in a neonatal unit: A systematic review. BMJ Open. 2016;6:e010466. https:// doi.org/10.1136/bmjopen-2015-010466

PMid:27797978

18. Onyedibe $\mathrm{KI}$, Utoh-Nedosa $\mathrm{AU}$, Okolo M, Ikenna OK, Ita OI, Udoh UA, et al. Impact of socioeconomic factors on neonatal sepsis in Jos, Nigeria. Jos J Med. 2012;6(2):54-8.

19. Hayun M, Alasiry E, Daud D, Febriani DB, Madjid D. The risk factors of early onset neonatal sepsis. Am J Clin Exp Med. 2015;3(3):78-82. https://doi.org/10.11648/j.ajcem.20150303.11

20. Casy BM, Mclntire DD, Leveno KJ. The continuing value of the Apgar score for the assessment of newborn infants.
N Engl J Med. 2001;344(7):467-71. https://doi.org/10.1056/ NEJM200102153440701

PMid: 11172187

21. Shah GS, Budhatoki S, Das BK, Mandal RN. Risk factors in early neonatal sepsis. Kathmandu Univ Med J (KUMJ). 2006;4(2):187-91.

PMid:18603896

22. Siakwa M, Kpikpitse D, Mupepi SC, Semuatu M. Neonatal sepsis in Rural Ghana: A case control study of risk factors in a birth cohort. Int J Res Med Health Sci. 2014;4(5):72-83.

23. Rozanska A, Wojkowsk-Mach J, Adamski P, BorszewskaKornacka M, Gulczyńska E, Nowiczewski M, et al. Infections and risk-adjusted length of stay and hospital mortality in Polish neonatology intensive care units. Int $\mathrm{J}$ Infect Dis. 2015;35:87-92. https://doi.org/10.1016/j.jij. 2015.04.017

PMid:25936583 